\title{
Emulation of Fading Wireless Link Effects in NEWS Wired Testbed
}

\author{
Homayoun Yousefi'zadeh Xiaolong Li \\ Department of EECS \\ University of California, Irvine \\ [hyousefi,xiaolonl] euci.edu
}

\author{
Wojtek Furmanski David B. Lofquist \\ Integrated Defense Systems \\ The Boeing Company \\ [wojtek.furmanski, david.b.lofquist] @boeing.com
}

\begin{abstract}
Currently under development by the Boeing Company, Network Emulator Wireless Simulator (NEWS) is a wired testbed for evaluating the performance of MANET protocols. In part, NEWS extends the Mobile Ad-Hoc Network Emulator (MANE) originally developed by Navy Research Lab (NRL). In this paper, we report the addition of emulation functionality to the NEWS testbed capturing fading wireless link effects. In order to emulate wireless link effects, we develop a finite-state discrete-time Markov chain the parameters of which are extracted from the fading, shadowing, transmission power, modulation type, and antenna configuration characteristics of the underlying link. We extend the implementation of MANE to include our Markov chain. We also investigate the effects of utilizing FEC techniques at the link layer in order to improve the performance of data delivery. Our numerical results show that ignoring the effects of fading can lead to inaccurate modeling of link effects in MANETs. Further, utilizing FEC techniques at the link layer can significantly improve the performance of data delivery over MANETs.

Index Terms-Ad-Hoc Networks, Fading Channel, Markov chain, MANE, Link Effects, Performance Evaluation.
\end{abstract}

\section{INTRODUCTION}

The need for developing wired testbeds to evaluate the performance of MANET protocols is well understood. Considering the time varying quality of wireless links utilized in MANETs, such testbeds are required to emulate wireless link effects in order to provide realistic wireless operation scenarios. Network Emulator Wireless Simulator (NEWS) is one such testbed currently under development by the Boeing Company. In part, NEWS extends emulation tools originally developed by Navy Research Lab (NRL) such as the Mobile Network Emulator (MNE) and the Mobile Ad-Hoc Network Emulator (MANE) [1]. Utilizing Linux firewalls, MNE emulates wireless link effects with a binary model representing either a high quality or a broken link. MANE offers support for capturing link effects relying on a distance based attenuation model.

Since neither MNE nor MANE account for temporally correlated effects in the behavior of the signals of interest,

This work was sponsored by grant no. COM06-10223 from Boeing Integrated Defense Systems and UC Discovery Industry-University Cooperative Research Program. it is important to add functionality related to the capturing of such effects in order to improve the accuracy of modeling. The main contribution of this paper is the addition of emulation functionality to the NEWS wired testbed -as an extension of MANE- offering effective means of performance evaluation for MANETs with fading wireless links. In order to emulate wireless link effects, a finite-state discrete-time Markov chain is developed with the parameters of the model extracted from fading, shadowing, transmission power, modulation type, and antenna configuration characteristics of the underlying fading wireless link.

The rest of this paper is organized as follows. Section II provides an in depth analysis of a fading wireless channel. It models the temporally correlated loss observed in a wireless channel with Markov chains. The section also explains how the use of Forward Error Correction (FEC) schemes at the link layer can combat the fading effects. Section III includes an overview of MANE as the building block of NEWS and how the model of Section II is used to revise the existing implementation of MANE. Section IV contains the results of our experiments and the related discussion. Finally, Section $\mathrm{V}$ concludes the paper.

\section{Wireless ChANNEL ANALYSIS}

This section provides an analysis of the wireless channel representing the characteristics of the underlying communication system. It includes a discussion related to the modeling of fading wireless links with finite-state Markov chains based on providing an analysis of Signal-to-InterferenceNoise Ratio $(S I N R)$ and calculating the Symbol Error Rate $(S E R)$ of such links. The latter captures the effects of utilizing multiple antennas on both transmitting and receiving ends.

\section{A. Analysis of SINR}

This section provides an analysis related to the extraction of the average values of $S I N R$ from the statistics of the fading channel, transmission powers, average noise power, shadowing, and in the presence of inter-symbol interference. Although the details of discussion below relate to rich scattering links characterized by Rayleigh fading, the approach 
is generic and can also be applied to the case of line of sight links characterized by Rician fading. As such, the discussion can be treated as a generalization of the discussions of [10] and [9].

Consider a number of wireless links, with subscript $i$ identifying the quantities associated with link $i$. Assume that the $M_{i} \times N_{i}$ link $i$ is equipped with $M_{i}$ transmit and $N_{i}$ receive antennas. The per symbol transmission power $P_{i}(t)$ is assumed to be fixed and equally distributed among $M_{i}$ transmit antennas. As the result of having $N_{i}$ receive antennas, the magnitude of noise in link $i$ is multiplied by $N_{i}$. The nonnegative number $G_{i j}(t)$ represents the path gain in the absence of fading from the transmitter of link $j$ to the receiver of link $i$ at time $t$. $G_{i j}(t)$ captures factors such as path loss, shadowing, antenna gain, and so on. Further, $F_{i j}^{(m, n)}(t)$ is the fading factor between transmitter $m$ of link $j$ and receiver $n$ of link $i$. The instantaneous signal-to-interference-noise ratio at time $t$ for link $i$, denoted by $S I N R_{i}(t)$, determines the quality of the received signal. Treating the fading distributions $F_{i i}^{(m, n)}$ as Identically and Independently Distributed (IID), $S I N R_{i}(t)$ is defined as

$$
\operatorname{SINR}_{i}(t)=\frac{G_{i i}(t) \frac{P_{i}(t)}{M_{i}} \sum_{m=1}^{M_{i}} \sum_{n=1}^{N_{i}} F_{i i}^{(m, n)}(t)}{\sum_{j \neq i} G_{i j}(t) \frac{P_{j}(t)}{M_{j}} \sum_{m=1}^{M_{j}} \sum_{n=1}^{N_{i}} F_{i j}^{(m, n)}(t)+N_{i} \eta_{i}(t)}
$$

where $\eta_{i}(t)$ represents the power of white Gaussian noise in link $i$.

In order to capture the temporal correlation of the wireless channel indicated in the equation above, few realistic assumptions are made as follows. First, the distribution of the white Gaussian noise is independent of the fading distributions. Second, the fading factors in interfering signals $F_{i j}$ 's are not spatially correlated. Third, $F_{i j}$ 's have unit means so long as $G_{i j}$ 's are appropriately scaled to reflect variations from this assumption. Fourth, when the wireless channel varies slowly with respect to symbol interval, $P_{i}(t)$ and $G_{i j}(t)$ can be viewed as constants and $F_{i j}(t)$ as a random variable within the symbol interval. In the rest of the discussion of this section, we work with the resulting random variables in the symbol interval.

From the discussion above, we need to examine the Probability Density Function (PDF) of random variables $F_{i j}$ and $\eta_{i}$ in order to obtain the PDF of the random variable $S I N R_{i}$. First, we list a number of properties of random variables from [6].

- Property 1: If $x$ and $y$ are random variables satisfying $y=g(x)$, then the PDF of $y$ satisfies $f_{y}(y)=\frac{f_{x}\left(x_{1}\right)}{g^{\prime}\left(x_{1}\right)}+$ $\cdots+\frac{f_{x}\left(x_{q}\right)}{g^{\prime}\left(x_{q}\right)}$ where $x_{1}, \cdots, x_{q}$ are the real roots of $y=$ $g(x)$ and $g^{\prime}(x)$ represents the derivative of $g(x)$.

- Property 2: If the PDF set $\left\{f_{x_{1}}\left(x_{1}\right), \cdots, f_{x_{q}}\left(x_{q}\right)\right\}$ is associated with the set of independent random variables $\left\{x_{1}, \cdots, x_{q}\right\}$, then the PDF of their sum $z=x_{1}+\cdots+$ $x_{q}$ is calculated as $f_{z}(z)=f_{x_{1}}\left(x_{1}\right) * \cdots * f_{x_{q}}\left(x_{q}\right)$ where $*$ represents the convolution operator.

- Property 3: If $x, y$, and $z$ are random variables satisfying $z=\frac{x}{y}$, the PDF of the random variable $z$ is specified as $f_{z}(z)=\int_{-\infty}^{\infty}|y| f_{x y}(y z, y) d y$.

Next, we focus on the identification of the PDF of $S I N R_{i}$. While we utilize the NLOS Rayleigh model with the fading factor $\alpha_{i}$ to relate the input and output of the path between transmit antenna $m$ and receive antenna $n$ of link $i$, it is possible to work with other models such as the LOS Rician model. We refer the reader to [9] for further details of utilizing Rician fading. The output signal of the Rayleigh channel $\Omega_{i}^{(m, n)}$ and its input signal $S_{i}^{(m, n)}$ over link $i$ can be related by

$$
\Omega_{i}^{(m, n)}=\alpha_{i} S_{i}^{(m, n)}+n_{i}
$$

where $n_{i}$ represents white Gaussian noise. It is well established [6] that $r_{i}=\left|\alpha_{i}\right|$ has a marginal Rayleigh PDF in the form of

$$
p_{R}\left(r_{i}\right)=\frac{r_{i} e^{-r_{i}^{2} / 2 \mu_{i}^{2}}}{\mu_{i}^{2}}, r_{i} \geq 0
$$

where $\mu_{i}^{2}$ equals to half of the average power of all of the multipath components. Since $F_{i i}^{(m, n)}$ is defined as $F_{i i}^{(m, n)}=$ $r_{i}^{2}=\left|\alpha_{i}\right|^{2}$, we are interested in the distribution of $\left|\alpha_{i}\right|^{2}$. The distribution of $\left|\alpha_{i}\right|^{2}$ can be calculated utilizing Equation (3) and Property 1 above. Thus,

$$
p_{F}\left(F_{i i}^{(m, n)}\right)=\frac{1}{2 \sqrt{F_{i i}^{(m, n)}}} p_{R}\left(\sqrt{F_{i i}^{(m, n)}}\right)
$$

We conclude that $F_{i i}^{(m, n)}$ has a marginal exponential PDF as

$$
p_{F}\left(F_{i i}^{(m, n)}\right)=\frac{e^{-F_{i i}^{(m, n)} / 2 \mu_{i}^{2}}}{2 \mu_{i}^{2}}, \quad F_{i i}^{(m, n)} \geq 0
$$

A similar argument is applied to the distribution of the random variables $F_{i j}^{(m, n)}$. Since the noise signal $n_{i}$ is assumed to be a complex random variable with its independent real and imaginary components having standard Gaussian distributions, its power $\eta_{i}$ has a Chi-square distribution with two degrees of freedom, or equivalently, an exponential distribution [6]. We note that while the fading distributions $F_{i j}^{(m, n)}$ and noise are considered to be independent, their parameters may not be necessarily the same.

Having identified the PDFs of $F_{i i}^{(m, n)}, F_{i j}^{(m, n)}$, and $\eta_{i}$, the PDF of $S I N R_{i}$ which is expressed as a function of the random variables above can be numerically identified relying on the properties of random variables described above. 


\section{B. Calculating Symbol Error Rates of Fading Wireless Links}

We now discuss how to calculate modulation symbol error rate $S E R_{i}$ of link $i$ in terms of the number of points in the modulation constellation, antenna configuration of the link, and average received $S I N R_{i}$ of the link.

In [8], the authors identify closed-form expressions describing the modulation symbol error rate of a multiple antenna wireless link $i$ in terms of the number of signal points in the constellation $M_{i}$ and the average received Signal-toNoise Ratio $(S N R)$ of link $i$ denoted by $S N R_{i}$. Calculations are carried out our under the assumption of facing a flat fading Rayleigh channel -implying that the channel is fixed in the duration of a symbol but varies from one symbol to another- and utilizing PSK modulation. We also note that the approach can be applied to other fading channels such as a Nakagami channel or other modulation schemes such as QAM. In what follows, we apply the results to capture the modulation symbol error rates of a wireless link. We note that we use the quantity $S I N R_{i}$ instead of $S N R_{i}$ in the current discussion.

First, we introduce the modulation symbol error rate of a link associated with single transmit and $N_{i}$ receive antenna nodes using Maximum Ratio Combining (MRC) as

$$
\begin{aligned}
& S E R_{i}=\frac{M_{i}-1}{M_{i}}-\frac{1}{\pi} \sqrt{\frac{\vartheta_{i}}{1+\vartheta_{i}}}\left\{\left(\frac{\pi}{2}+\tan ^{-1} \xi_{i}\right)\right. \\
& \sum_{p=0}^{N_{i}-1}\left(\begin{array}{c}
2 p \\
p
\end{array}\right) \frac{1}{\left[4\left(1+\vartheta_{i}\right)\right]^{p}}+\sin \left(\tan ^{-1} \xi_{i}\right) \\
& \left.\sum_{p=1}^{N_{i}-1} \sum_{i=1}^{p} \frac{\sigma_{q p}}{\left(1+\vartheta_{i}\right)^{p}}\left[\cos \left(\tan ^{-1} \xi_{i}\right)\right]^{2(p-q)+1}\right\}
\end{aligned}
$$

where $\vartheta_{i}=S I N R_{i} \sin ^{2}\left(\frac{\pi}{M_{i}}\right), \xi_{i}=\sqrt{\frac{\vartheta_{i}}{1+\vartheta_{i}}} \cot \frac{\pi}{M_{i}}$, and $\sigma_{q p}=\frac{\left(\begin{array}{c}2 p \\ p\end{array}\right)}{\left(\begin{array}{c}2(p-q) \\ p-q\end{array}\right) 4^{q}[2(p-q)+1]}$. From Equation (6), one can calculate the modulation symbol error rate of a link associated with single transmit single receive antenna nodes as well as a link associated with single transmit double receive antenna nodes by setting $N_{i}$ to 1 and 2 , respectively. Relying on a discussion of diversity gains and under the assumption that the transmission power is equally divided among the transmit antennas, we argue that the modulation symbol error rate of Space-Time Block Codes (STBCs) of [2] and [7] can be calculated from Equation (6) by proper scaling of the values of $S I N R_{i}$. For example, the modulation symbol error rate of a $2 \times 1$ link associated with can be calculated by replacing $S I N R_{i}$ with $\frac{S I N R_{i}}{2}$ and setting $N_{i}$ to 2 in Equation (6). Similarly, the modulation symbol error rate of a $2 \times 2$ link can be calculated by replacing $S I N R_{i}$ with $\frac{S I N R_{i}}{2}$ and setting $N_{i}$ to 4 in Equation (6). Based on the expressions above and similar to the previous section, the PDF of $S E R_{i}$ expressed as a function of $S I N R_{i}$ can be numerically identified.

\section{Markov Chain Modeling of Fading Wireless Links}

Having specified the PDFs of the signals of interest, we can now focus on capturing temporally correlated loss between Units of Information (UoIs) ${ }^{1}$ in a fading channel. In the discussion below and consistent with the notation of previous sections, subscript $i$ identifies the quantity of interest in link $i$.

While we direct our discussion to the case of a UoI represented by the symbol error rate in a Rayleigh fading channel, our discussion can be generalized to the case of any other UoI in any other fading channel. We propose modeling the temporally correlated symbol loss of an analog signal with an $S$-state digital Markov chain by means of partitioning its PDF. An $S$-state Markov chain is fully described by a set of transitioning probabilities between any pair of states and another set of per state $S E R_{i}$ probabilities. Considering the issue of practicality, our approach for extracting the two set of probabilities modifies the proposals of [10] and [9]. It is described below.

Although the number of states $S$ can be arbitrarily chosen, we set $S=2$ in order to address the tradeoff between lowering the complexity of calculations and improving the accuracy of the model. We note that our choice of $S=2$ reduces the $S$-state Markov chain model to the Gilbert-Elliott (GE) model [4] and [3]. Fig. 1 illustrates the modeling approach by means of partitioning the PDF of an arbitrary $S E R_{i}$ signal.
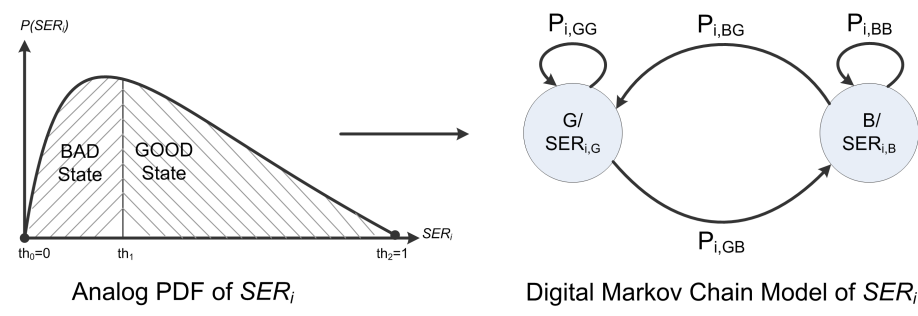

Digital Markov Chain Model of SER

Fig. 1. An illustration of Markov chain modeling for an arbitrary PDF associated with an analog $S E R_{i}$ signal.

The two-state GE loss model provides an elegant mathematical model to capture the symbol loss behavior. The GE model consists of a GOOD and a BAD state. The GOOD state introduces a probability $P_{i, G G}=\gamma_{i}$ of staying in the GOOD state and a probability $P_{i, G B}=1-\gamma_{i}$ of transitioning to the BAD state while the BAD state introduces a probability $P_{i, B B}=\beta_{i}$ of staying in the BAD state and a probability $P_{i, B G}=1-\beta_{i}$ of transitioning to the GOOD state. The GOOD state also represents average per state values $S E R_{i, B}$ and $S E R_{i, G}$ where $S E R_{i, B}>>S E R_{i, G}$.

The parameters $\gamma_{i}$ and $\beta_{i}$ can be measured from the observed average burst lengths of the arriving symbols [5]

\footnotetext{
${ }^{1} \mathrm{~A}$ UoI can be thought of as a bit, symbol, or packet.
} 
where a burst length is defined as the number of consecutive symbols arrived in the same state. Calling the average burst lengths of the BAD and GOOD state $\overline{B L}_{i, B}$ and $\overline{B L}_{i, G}$, the parameters $\gamma_{i}$ and $\beta_{i}$ are identified from the equations below.

$$
\overline{B L}_{i, B}=\frac{1}{1-\beta_{i}}, \quad \overline{B L}_{i, G}=\frac{1}{1-\gamma_{i}}
$$

Once $\gamma_{i}$ and $\beta_{i}$ are identified, the steady-state values of being in the GOOD state $g_{i, s s}$ and the BAD state $b_{i, s s}$ can be identified as

$$
b_{i, s s}=\frac{1-\gamma_{i}}{2-\gamma_{i}-\beta_{i}}, \quad g_{i, s s}=\frac{1-\beta_{i}}{2-\gamma_{i}-\beta_{i}}
$$

Referencing Fig. $1, b_{i, s s}$ and $g_{i, s s}$ also represent areas of the PDF limited between consecutive partitioning thresholds as

$$
\begin{aligned}
b_{i, s s} & =\int_{0}^{t h_{1}} p\left(S E R_{i}\right) d S E R_{i} \\
g_{i, s s} & =\int_{t h_{1}}^{1} p\left(S E R_{i}\right) d S E R_{i}
\end{aligned}
$$

Setting $t h_{0}=0$ and $t h_{2}=1$, the value of non-trivial threshold $t h_{1}$ can be numerically identified. Once the threshold values are identified, the quantities $S E R_{i, B}$ and $S E R_{i, G}$ are calculated as

$$
\begin{aligned}
& S E R_{i, B}=\int_{0}^{t h_{1}} S E R_{i} p\left(S E R_{i}\right) d S E R_{i} \\
& S E R_{i, G}=\int_{t h_{1}}^{1} S E R_{i} p\left(S E R_{i}\right) d S E R_{i}
\end{aligned}
$$

Let $\varphi_{i}(t, r, G)$ and $\varphi_{i}(t, r, B)$ denote the probability of receiving $r$ symbols from $t$ transmitted symbols and winding up in the GOOD and the BAD state of the GE model, respectively. Then the overall probability of receiving $r$ symbols from $t$ transmitted symbols for the GE model is given by

$$
\varphi_{i}(t, r)=\varphi_{i}(t, r, G)+\varphi_{i}(t, r, B)
$$

where the recursive probabilities $\varphi_{i}(t, r, G)$ and $\varphi_{i}(t, r, B)$ are given [10] by

$$
\begin{aligned}
& \varphi_{i}(t, r, G)= \\
& \quad S E R_{i, G}\left[\gamma_{i} \varphi_{i}(t-1, r, G)+\left(1-\beta_{i}\right) \varphi_{i}(t-1, r, B)\right] \\
& \quad\left(1-S E R_{i, G}\right)\left[\gamma_{i} \varphi_{i}(t-1, r-1, G)\right. \\
& \left.\quad+\left(1-\beta_{i}\right) \varphi_{i}(t-1, r-1, B)\right]
\end{aligned}
$$

and

$$
\begin{aligned}
& \varphi_{i}(t, r, B)= \\
& \quad S E R_{i, B}\left[\left(1-\gamma_{i}\right) \varphi_{i}(t-1, r, G)+\beta_{i} \varphi_{i}(t-1, r, B)\right] \\
& \left(1-S E R_{i, B}\right)\left[\left(1-\gamma_{i}\right) \varphi_{i}(t-1, r-1, G)\right. \\
& \left.\quad+\beta_{i} \varphi_{i}(t-1, r-1, B)\right]
\end{aligned}
$$

for $t \geq r>0$ and the initial conditions

$$
\begin{aligned}
\varphi_{i}(0,0, G) & =g_{i, s s}=\frac{1-\beta_{i}}{2-\gamma_{i}-\beta_{i}} \\
\varphi_{i}(0,0, B) & =b_{i, s s}=\frac{1-\gamma_{i}}{2-\gamma_{i}-\beta_{i}} \\
\varphi_{i}(1,0, G) & =S E R_{i, G}\left[\gamma_{i} g_{i, s s}+\left(1-\beta_{i}\right) b_{i, s s}\right] \\
\varphi_{i}(1,0, B) & =S E R_{i, B}\left[\left(1-\gamma_{i}\right) g_{i, s s}+\beta_{i} b_{i, s s}\right]
\end{aligned}
$$

\section{FEC for Fading Wireless Links}

In this section, we propose the use of the Reed-Solomon (RS) channel coder at the link layer as an optional component to compensate for temporally correlated loss effects observed over the wireless link. We note that an RS channel coder $R S(b, k)$ converts $k$ channel coding symbols into a $b$ symbol block by appending $(b-k)$ parity symbols ${ }^{2}$. Such channel coder is able to correct as many as $t_{C}=\left\lfloor\frac{b-k}{2}\right\rfloor$ channel coding symbol errors in a block. If at least $b-t_{C}$ channel coding symbols are correctly received from $b$ transmitted channel coding symbols, the whole block is recoverable.

Suppose the RS coder generates a set of channel coding symbols where each symbol consists of $s$ bits. A channel coding symbol is received error free if all of its $s$ bits are received free of errors. As indicated earlier, we assume that the loss pattern of the wireless channel at the bit level is described by the GE model. Thus, the probability of receiving a channel coding symbol free of error and winding up in the GOOD state is obtained from Equation (12) with $t=r=s$ as $\varphi(s, s, G)$. Similarly, the probability of receiving a channel coding symbol free of error and winding up in the BAD state is obtained from Equation (13) as $\varphi(s, s, G)$.

Since the inter-symbol temporal correlation has to be captured for back-to-back transmitted channel coding symbols over the same GE model with the same state transitioning probabilities, the probability of channel coding symbol block loss is obtained utilizing Equation (11), (12), and (13) as

$$
\Psi\left(b, t_{C}, \chi_{G}, \chi_{B}\right)=\sum_{i=b-t_{C}}^{b} \varphi(b, i)
$$

where $\chi_{G}$ and $\chi_{B}$ identified below play the roles of $S E R_{i, G}$ and $S E R_{i, B}$ in equations (12) and (13).

$$
\begin{aligned}
& \chi_{G}=1-\varphi(s, s, G) \\
& \chi_{B}=1-\varphi(s, s, B)
\end{aligned}
$$

The initial conditions are identified from Equation (14) with the same setting of parameters as the ones indicated in Equation (16).

\section{REVISING MANE'S IMPLEMENTATION FOR NEWS}

In this section, we briefly describe the functionality of MANE [1] as the building block of NEWS and explain how NEWS revises the current implementation of MANE based on the discussion of the previous section.

\footnotetext{
${ }^{2}$ We distinguish between the modulation symbols and channel coding symbols by noting that a channel coding symbol typically consists of a number of modulation symbols. For example, an 8-bit RS channel coding symbol consists of eight BPSK modulation symbols.
} 
As illustrated by Fig. 2, MANE is a software-hardware testbed tool used to emulate a MANET environment for a number of Test Nodes (TNs) arranged in a number of clusters with activity in each cluster supervised by a MANE Server (MS). The testbed can potentially consist of multiple clusters. In a multiple cluster testbed, MANE servers are interconnected via two LANs; one LAN is used as a control bus while the other is used as a data bus.

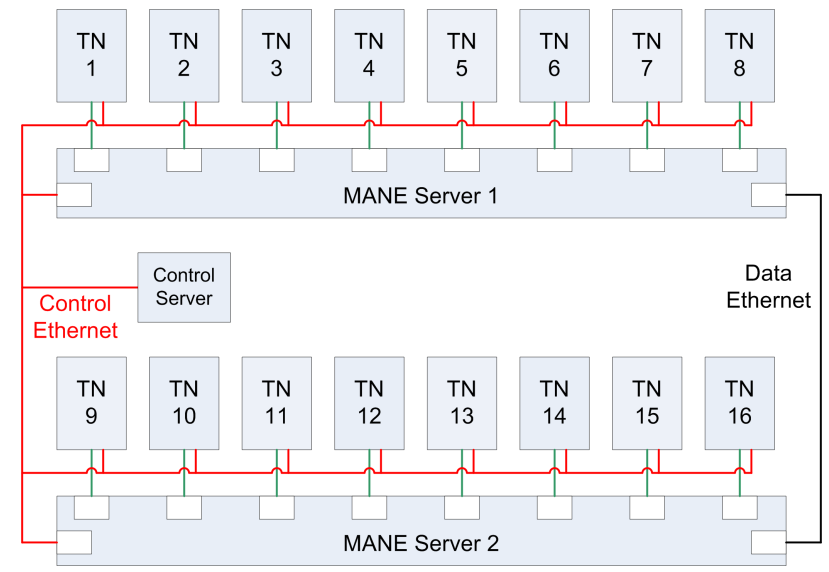

Fig. 2. A schematic block diagram of a multiple cluster MANE testbed.

As observed from Fig. 2, each TN has a point-to-point connection to its cluster MS. The exchange of data traffic between TNs is done utilizing the point-to-point connections via a single or multiple MS interfaces depending on whether or not the TNs belong to the same cluster. Flowing data traffic through MS servers allows for offering a capability to enforce wireless topology control and link effect emulation.

MANE consists of six major software components hosted in Linux Operating System user and kernel spaces. The first three components are hosted in Linux application space and run on MANE servers. They include: (1) the forwarding engine component that is responsible for forwarding packets between TNs and can drop packets based upon information provided by MANE's second component referred to as the range model, (2) the range model component that is responsible for calculating the probability of dropping a packet and (3) the Global Positioning System (GPS) emulation component that is responsible for providing other components of the system with GPS information.

The next three components run on MANE TNs and include: (1) the GPS daemon component that is running in Linux application space and responsible for delivering GPS information to the end users, (2) the TNTuneling component that is running in Linux application space and responsible for limiting traffic coming from or going to TNs by utilizing a virtual interface capable of emulating Medium Access Control (MAC) effects, and (3) the tunNRL Loadable Kernel Module (LKM) component that is running in Linux kernel space and supports the operation of TNTuneling component.

As indicated above, MANE relies on the range model to emulate the link effects. The current implementation of the range model utilizes a reference model to create a table of values of Bit Error Rate $(B E R)$ versus $S N R$ for a given modulation scheme. The reference model of the range model is somewhat simplistic because the calculation of $S N R$ only considers the transmit power of the desired signal, the average power of noise, and a signal attenuation solely based on distance leaving out the effects of fading as well as interference from other nodes, antenna configuration, and channel coding. Consequently, no temporal correlation is captured in the loss of multiple bits either. More specifically, the range model calculates the average $S N R_{i}$ of a link $i$ as

$$
S N R_{i}=\frac{P_{i} G_{i i}}{\eta_{i}}
$$

where the fixed value of $G_{i i}$ in the symbol interval is inversely proportional with the third power of distance between the link end nodes. Relying on the value of $S N R_{i}$, the range model calculates the bit error rate $B E R_{i}$ of link $i$ as

$$
B E R_{i}=\frac{1}{2} Q\left(\sqrt{m_{i} S N R_{i}}\right)
$$

where $m_{i}$ is a fixed factor uniquely mapped to the number of points in modulation constellation and the Gaussian $Q$ function is defined as

$$
Q(x)=\frac{1}{\sqrt{2 \pi}} \int_{x}^{\infty} \exp \left(-\frac{z^{2}}{2}\right) d z
$$

Finally, MANE treats a byte as an atomic UoI which can also be thought of the channel coding symbol size allowing a byte to be received only if all of its eight bits are received free of errors. For BPSK modulation, MANE calculates the probability of byte loss $\varepsilon_{i}$ for link $i$ utilizing a memoryless model as

$$
\varepsilon_{i}=1-\left(1-B E R_{i}\right)^{8}
$$

Since the shortcomings of the implementation closely relate to the reality of wireless links, it is important that the current implementation of MANE is modified by NEWS such that the revised model addresses the shortcomings indicated above. NEWS modifies the current implementation of MANE relying on the assumption that the loss pattern of the wireless channel at the bit level is described by the GE model. Thus, the probability of byte loss under the GE model is calculated as

$$
\varepsilon_{i}=1-\varphi_{i}(8,8)
$$

where $\varphi_{i}(8,8)$ is identified by Equation (11) and subsequently Equation (12) and Equation (13) by setting $t=r=$ 8. Further, the quantities $S E R_{i, B}$ and $S E R_{i, G}$ are specified 
utilizing Equation (10). We note that the approach of NEWS allows for identifying the probability of byte loss utilizing QPSK and 8-PSK by calculating the probability of losing two and one symbols, respectively.

At the end of this section, the follwoing statements are in order regarding the the link effect emulation capability of NEWS. We note that MANE utilizes multiple clusters in which the bulk of the processing work is handled by MANE servers and in the user space of Linux operating system as oppose to the kernel space of Linux operating system. However, we have developed a decentralized model for emulating the link effects cosnidering the high processing overhead of MANE. Our model implements the finitie-state discretetime Markov chain associated with a link utilizing a Linux loadable kernel module running at the receiving side of the link. The parameters of the chain are delivered to the driver through a system call in the Linux user space. An application makes such a system call after extracting the parameters of the model from the fading, shadowing, transmission power, modulation type, and antenna configuration characteristics.

\section{EXPERIMENTAL RESULTS}

In this section, we describe the performance evaluation results of the implementation of NEWS.

For our experiments, we utilize the diamond MANET topology of Fig. 3. The network consists of wireless nodes $N_{1}, N_{2}, N_{3}$, and $N_{4}$ along with 4 links $\mathcal{L}_{1}$ through $\mathcal{L}_{4}$. Nodes $N_{1}$ and $N_{4}$ are assumed to be parts of two platforms $A$ and $B$ accommodating wired links. Nodes $N_{1}$ and $N_{4}$ are assumed to act as the wireless gateway of their associated platform. Communication over fading links is assumed to be subject to interference caused by transmission associated with other links in addition to noise. We assume that each fading wireless channel is characterized by a Rayleigh distribution with fading factors that are constant over a symbol but vary independently from one symbol to another.

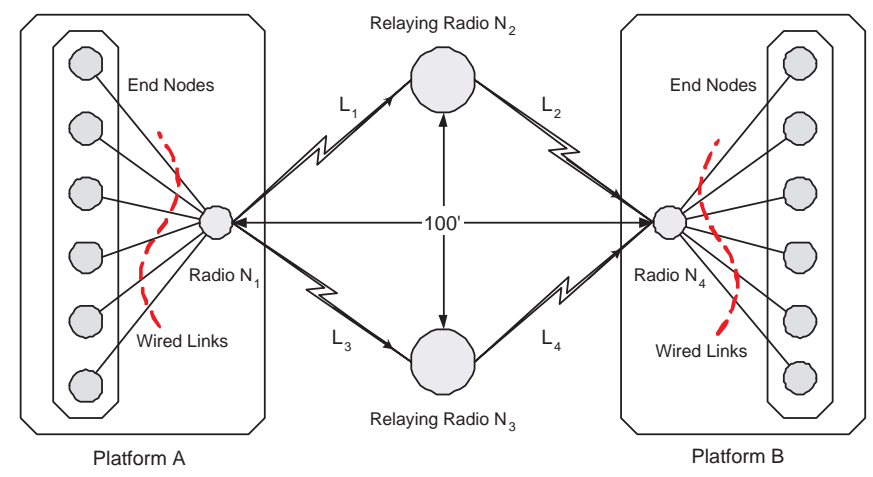

Fig. 3. An illustration of the MANET topology used in performance evaluation experiments.
In the context of NEWS, the topology is mapped to using 4TNs in a single cluster NEWS setting. We consider four different scenarios: (1) each radio node has a single antenna, (2) $N_{1}$ has double antennas and the rest of radio nodes have a single antenna, (3) $N_{2}$ and $N_{3}$ have double antennas and the other two radio nodes have a single antenna, and (4) all of the radio nodes have double antennas. Each $1 \times 2$ link utilizes MRC and each $2 \times 1$ or $2 \times 2$ link utilizes STBCs of [7].

The followings represent various settings of the wireless channel for the reported results. First, the transmission range of $N_{1}$ on link $\mathcal{L}_{1}$ is $[0.1 \mathrm{~W}, 5 \mathrm{~W}]$ with the transmission powers on other links fixed at $1 \mathrm{~W}$ and the average power of noise fixed at $1 \mu \mathrm{W}$. With the exception of $G_{12}$ and $G_{34}$, the gains for each link are computed as $G_{i i}=\frac{1}{d_{i i}^{2}}$ and $G_{i j}=\frac{0.005}{d_{i j}^{2}}$ for $i \neq j$ where $d_{i j}$ represents propagation path length from the transmitter of link $j$ to the receiver of link $i$. The gains for $G_{12}$ and $G_{34}$ are set to zero since we assume a node cannot transmit and receive at the same time. All nodes are using BPSK modulation.

We set transition probabilities of the GE model for a $1 \times 1$ link as $\gamma=0.995$ and $\beta=0.95$ represented by average burst lengths of 500 and 20 bits for the GOOD and BAD state, respectively. Using the values of $\gamma$ and $\beta$, we numerically calculate the non-trivial threshold of partitioning $t h_{1}$ for the PDF of $S E R_{i}$ over the $1 \times 1$ link of study. Then, we use Equation (10) to identify $S E R_{i, G}$ and $S E R_{i, B}$ for the link. Finally, we use Equation (6) to map deterministic values of $S E R_{i, G}$ and $S E R_{i, B}$ to $S I N R_{i, G}$ and $S I N R_{i, B}$ for the link. We note that the use of transmission range $[0.1 \mathrm{~W}, 5 \mathrm{~W}]$ allows for achieving a range of $[0,50] d B$ for the quantity $S I N R_{1, G}$ with associated values for the quantity $S I N R_{1, B}$.

We select $6 \mathrm{ftp}$ sources in Platform A to send packets to destinations in Platform B. Each ftp session attempts at delivering a $1 \mathrm{MB}$ file. We rely on the standard implementation of TCP with Random Early Drop (RED) in Linux Kernel 2.6.15 to establish end-to-end ftp sessions. While the capacity and the delay of the wireless links are assumed to be $10 \mathrm{Mbps}$ and $200 \mathrm{msec}$, the wired links have a capacity of $1 \mathrm{Gbps}$ and a delay of $6.4 \mathrm{msec}$. All of the links operate in a full-duplex mode and link bandwidths are shared among data and acknowledgment (ACK) packets. Each packet is treated as a channel coding block with a symbol size of one byte. We allocate a small percentage of the overall bandwidth to FEC, i.e., $1.5 \%$ of the space in each data packet of size 1040 bytes and $50 \%$ of the space in each ACK packet of size 40 bytes. Such allocation has significant performance improvement effects.

In the following curves, the horizontal axis captures the deterministic variation range of $S I N R_{1, G}$ over link $\mathcal{L}_{1}$ as an indicator of the link quality. The deterministic range of $S I N R_{G}$ (and $S I N R_{1, B}$ not shown) is derived from the de- 
terministic range of $S E R_{1, G}\left(S E R_{1, B}\right)$ calculated for the GE model relying on Equation (6). The GE model parameters of other links are fixed assuming values proportional to one of the four scenarios of operation mentioned above.

Averaged over all six flows, Fig. 4 compares the end-toend Packet Error Rate (PER) of the four antenna configuration scenarios mentioned above. As illustrated, all four curves exhibit saturation-like behavior directly related to the qualities of wireless links. For small values of $S I N R_{1, G}$ representing a low link quality, the value of average PER is close to $50 \%$. As the quality of the link $\mathcal{L}_{1}$ improves, the value of PER improves eventually approaching zero. When comparing the results of different antenna configurations, we observe that the performance of scenarios (1), (2), (3), and (4) are in an ascending order as the result of improving the quality of multiple antenna links. We make note of the poor quality of transmission for the low values of $S I N R_{1, G}$ in all four curves that would not be captured without proper modeling of temporally correlated loss of the links. Fig. 5

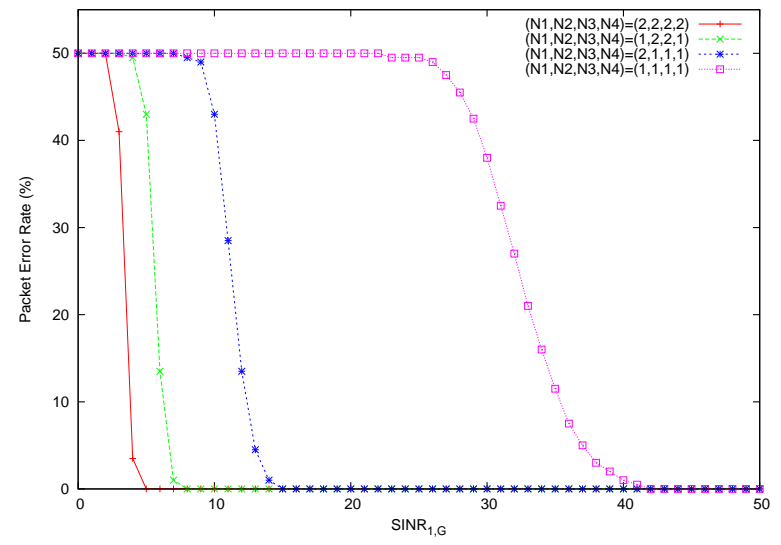

Fig. 4. A comparison of average Packet Error Rates for four scenarios in which wireless radio nodes $\left(N_{1}, N_{2}, N_{3}, N_{4}\right)$ are equipped with a number of antennas indicated in the captions.

compares the average ftp completion time of the four scenarios mentioned above. It is important to note that the starting points of the curves correspond to experiments that can finish in a finite period of time, i.e., cases in which large values of PER for link $\mathcal{L}_{1}$ does not allow the completion of ftp sessions are not shown. Once more, it is observed that increasing the number of multiple antenna links improves the performance measure.

\section{CONCLUding REMARKS}

In this paper, we reported the results of a link effect emulation capability for a MANET wired testbed called Network Emulator Wireless Simulator (NEWS) currently under development by the Boeing Company. We noted that, NEWS extends the Mobile Ad-Hoc Network Emulator (MANE) originally developed by Navy Research Lab (NRL). To address

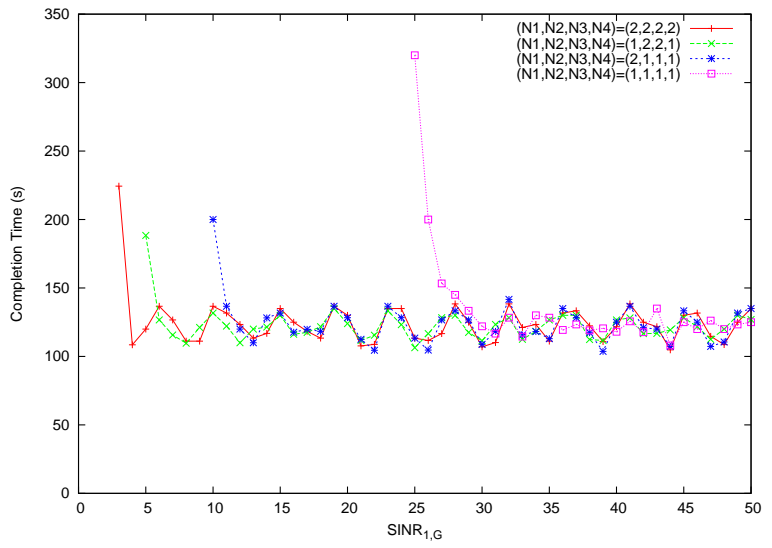

Fig. 5. A comparison of ftp completion times for four scenarios in which wireless radio nodes $\left(N_{1}, N_{2}, N_{3}, N_{4}\right)$ are equipped with a number of antennas indicated in the captions.

the shortcomings of MANE's distance-based link effect emulator and in order to capture fading wireless link effects, we reported the addition of a wireless link effect emulation functionality to the NEWS testbed. We reported the results of our recent development work utilizing finite-state Markov chains the parameters of which were extracted from the fading, shadowing, transmission power, modulation type, and antenna configuration characteristics of an underlying fading wireless link. Our numerical results showed that ignoring the effects of fading can lead to inaccurate modeling of link effects in MANETs.

\section{REFERENCES}

[1] -, "Mobile Ad-hoc Network Emulator," Navy Research Labs, Available at http://cs.itd.nrl.navy.mil/work/mane

[2] S.M. Alamouti, "A Simple Transmitter Diversity Scheme for Wireless Communications," IEEE JSAC, November 1998.

[3] E.O. Elliott, "Estimates on Error Rates for Codes on Burst-Noise Channels," Bell Syst. Tech. J., September 1963.

[4] E.N. Gilbert, "Capacity of A Burst-Noise Channel," Bell Syst. Tech J., September 1960.

[5] H. Jafarkhani, P. Ligdas, N. Farvardin, "Adaptive Rate Allocation in a Joint Source-Channel Coding Framework for Wireless Channels," In Proc. IEEE VTC, 1996.

[6] A. Papoulis, S.U. Pillai, "Probability, Random Variables, and Stochastic Processes, Fourth Edition," McGraw-Hill, ISBN $0071122567,2002$.

[7] V. Tarokh, H. Jafarkhani, A.R. Calderbank, "Space-Time Block Coding from Orthogonal Designs," IEEE Trans. Information Theory, July 1999.

[8] H. Yousefi'zadeh, H. Jafarkhani, M. Moshfeghi, "Power Optimization of Wireless Media Systems with Space-Time Block Codes," IEEE Trans. Image Processing, July 2004.

[9] H. Yousefi'zadeh, "A Constrained Resource Allocation Study for LOS MIMO Fading Ad-Hoc Networks," In Proc. IEEE WCNC, 2006.

[10] L. Zheng, H. Yousefi'zadeh, H. Jafarkhani, "Resource Allocation in Fading Wireless Ad-Hoc Networks with Temporally Correlated Loss," In Proc. of IEEE WCNC, 2004. 